\title{
Zwischen traditioneller und moderner Medizin? Internationale Studierende diskutieren Heraus- forderungen in der weltweiten Gesundheitsversorgung
}

STUBE Hessen ist ein studienbegleitendes entwicklungspolitisches Programm für internationale Studierende in Hessen und ein Projekt des World University Service (WUS), gefördert vom Hessischen Ministerium für Wissenschaft und Kunst. Die Studierenden erarbeiten die Themen, die bei STUBE behandelt werden, in einem partizipativen Verfahren. Die von ihnen vorgeschlagenen Veranstaltungsthemen waren und sind auch weiterhin von Fragen geprägt, wie eine verlässliche Gesundheitsversorgung für alle Menschen zur globalen Wirklichkeit werden kann und welche gesundheitlichen Herausforderungen sich die Menschheit gegenwärtig ausgesetzt sieht. Eines der gewählten Themen für das Jahr 2021 ist „Pflege weltweit - die vergessenen Kranken“ und wurde im Mai 2021 in Form eines Wochenendseminars angeboten. Nach Berechnungen der Weltgesundheitsorganisation (WHO) fehlen weltweit über 17 Millionen Gesundheitsfachkräfte, davon 2,6 Millionen Ärzt/-innen sowie 9 Millionen Krankenpfleger/-innen und Hebamm/-innen. Mehr als $80 \%$ der Pflegekräfte arbeiten in Ländern, in denen die Hälfte der Weltbevölkerung lebt. Im Rahmen des Seminars beleuchten die Teilnehmenden diese Ungleichheit gemeinsam mit Referent/-innen aus Wissenschaft und Zivilgesellschaft.

Bei STUBE entwickeln die Studierenden mögliche Lösungswege, um zu einer sozial und ökologisch nachhaltigen Entwicklung in ihren Herkunftsländern und in Hessen beizutragen. Neben den siebzehn Nachhaltigkeitszielen der Vereinten Nationen (Sustainable Development Goals, SDG) steht der Wissensaustausch von Studierenden untereinander, aber auch mit Lehrenden aus Ländern des Globalen Südens und des Nordens im Zentrum des Studienbegleitprogramms. So referierte Kolahta Asres Ioab, Studentin der Hochschule Fulda aus Eritrea, im Rahmen eines virtuellen Workshops von STUBE und academic experience Worldwide e.V. zum Thema „Migration and the COVID-19-Pandemic“. Die Frage, wie sich die Pandemie auf etablierte Gesundheitssysteme und bestehende Ungerechtigkeiten auswirkt, diskutierte sie gemeinsam mit internationalen Studierenden und Geflüchteten im März 2021. Im Herbst 2020 fand die fünftägige STUBE-Akademie „Gesundheitsversorgung weltweit: Moderne vs. Traditionelle Medizin“ statt. Hier verdeutlichte Dr. Sascha Krannich, wissenschaftlicher Mitarbeiter am Institut für Geschichte der Medizin an der Justus-Liebig-Universität, dass „Gesundheit ist ein Menschenrecht!“ nach wie vor eine normative Forderung statt eines Ist-Zustands beschreibt. Und das, obwohl die WHO bereits im September 1978 in Alma-Ata „Health for All - Gesundheit für alle“ deklarierte. Wie das dritte Ziel der SDG, ein gesundes Leben für alle Menschen jeden Alters zu gewährleisten und ihr Wohlergehen zu fördern, bis zum Jahr 2030 erreicht werden kann, erörterten die Teilnehmenden im weiteren Akademieverlauf. Am Beispiel der Bilharziose ging Antonia Asche, Fachreferentin des Unternehmens Merck KGaA, in ihrem Workshop auf die Herausforderungen in der Bekämpfung von vernachlässigten Tropenkrankheiten ein. Nur wenige Men- schen sind mit den Risiken der Bilharziose vertraut, auch bekannt als Schistosomiasis, obwohl fast 240 Millionen Menschen in 78 Ländern Afrikas, Asiens und Südamerikas von der Krankheit betroffen sind. Ein Mensch infiziert sich mit Bilharziose, wenn er mit Wasserquellen in Kontakt kommt, die durch eine bestimmte Art von freischwimmenden Parasiten verunreinigt sind.

Janak Dhakal aus Nepal, Medizinstudent der Goethe-Universität Frankfurt a. M., gab den Teilnehmenden in seinem Workshop „Generika: Pharmaindustrie in der Krise?“ mithilfe der digitalen Werkzeuge Kahoot und mentimeter einen Überblick über Kriterien, entlang welcher sich Gesundheitssysteme in ihrer Qualität bewerten lassen, z.B. entlang des Anteils der laufenden Gesundheitsausgaben am Bruttoinlandsprodukt (BIP), der Anzahl von Krankenhausbetten, der Anzahl von Ärzt/-innen und Pflegepersonal sowie der durchschnittlichen Lebenserwartung. Anhand der Diskussion um Kopien von Medikamenten mit gleichem Wirkstoff, sogenannte Generika, hinterfragten die Studierenden, inwieweit der Patentschutz im Pharmabereich negative Auswirkungen auf die Versorgung der Bevölkerung in Ländern des Globalen Südens mit lebenswichtigen Medikamenten hat. Einen Ausblick auf zukünftige Entwicklungen und Innovationen in der medizinischen Versorgung gab Dupleix Achille Takoulegha aus Kamerun vom Fachbereich Gesundheit der Technischen Hochschule Mittelhessen (THM) unter dem Motto „Gesundheitsversorgung der Zukunft? E-Health und Telemedizin“. Telemedizin ist die Anwendung moderner Informations- und Kommunikationstechnologien im Gesundheitswesen, um konkrete medizinische Dienstleistungen in Überwindung räumlicher Entfernungen zu erbringen. Im Workshop von Prof. Dr. Dr. Reimer Gronemeyer, Ehrensenator der Justus-Liebig-Universität Gießen, beschäftigten sich die Studierenden mit der Frage, warum westliche Gesundheitssysteme im südlichen Afrika scheitern. Gronemeyer betonte, dass sich die Menschheit darüber im Klaren sein muss, dass das Wissen europäischer Pharmakonzerne zur Erlangung des genetischen Materials aus Afrika, Asien und Lateinamerika auch durch Raub nach Europa kam. Es gehe letztlich auch um die Anerkennung, dass Menschen vor vielen Jahrtausenden bereits sehr viel wussten und dieses Wissen dürfe nicht vergessen werden.

Um einige Erfahrungen und Perspektiven reicher, gab Kolahta Asres Ioab, die auch an der Herbstakademie teilnahm, abschließend zu denken, dass sich die Menschheit unter dem Stichwort „Planetary Health“ mit den Zusammenhängen zwischen der menschlichen Gesundheit und den politischen, ökonomischen und sozialen Systemen, sowie den natürlichen Systemen unseres Planeten, vertraut machen muss, um auch planetarische Veränderungen und deren Auswirkungen auf Gesundheit zu berücksichtigen.

Eileen Paßlack, World University Service Deutsches Komitee e. V. doi.org/10.31244/zep.2021.03.09 